DOI: 10.14451/1.167.12

\title{
ИННОВАЦИИ И ИХ РОЛЬ В ФОРМИРОВАНИИ МУЛЬТИПЛИКАТИВНЫХ ЭФФЕКТОВ
}

\author{
(c) 2018 Еремин Владимир Владимирович \\ кандидат экономических наук \\ ведущий научный сотрудник Центра стратегического прогнозирования и планирования \\ Институт экономической политики и проблем экономической безопасности \\ Финансовый университет при Правительстве Российской Федерации \\ 125993, г. Москва, Ленинградский пр-т, д. 49 \\ E-mail: villy.eremin@gmail.com. \\ (c) 2018 Котова Наталья Евгеньевна \\ младший научный сотрудник Центра макроэкономических исследований \\ Департамент экономической теории \\ Финансовый университет при Правительстве Российской Федерации \\ 125993, г. Москва, Ленинградский пр-т, д. 49 \\ E-mail:nkotova@fa.ru
}

Предметом статьи является исследование мультипликативных эффектов, генерируемых процессом развития инновационного сектора экономики. Влияние мультипликативных эффектов, формируемых инвестициями, направленными на развитие инновационного сектора, усложняет процесс его развития. Действие мультипликативных эффектов способно вызвать нехватку ресурсов, инфляцию. Что затормозит развитие как инновационного сектора, так и экономики в целом. Следовательно, развитие инновационного сектора экономики оказывает двоякое влияние на отечественную экономику. С одной стороны, оно усиливает экономическую безопасность, ослабляя зависимость отечественной экономики от иностранных технологий и инновационных продуктов. С другой стороны - ухудшает ситуацию с экономической безопасностью в результате негативного действия мультипликативных эффектов. Цель статьи - описать влияние мультипликативных эффектов, генерируемых развитием инновационного сектора на сам процесс этого развития, а также разработать методику, позволяющую оценить величину генерируемых мультипликативных эффектов. Оценка их величины и направлений распространения позволит разработать методы управления ими. Что позволит минимизировать их негативное влияние как на экономические процессы в целом, так и на развитие инновационного сектора в частности. Процесс построения инновационной экономики станет более эффективным.

Результаты исследования могут применяться в рамках управления развитием инновационных секторов экономики Российской Федерации, в целях стабилизации этого развития, повышения его эффективности и снижения риска путем получения более предсказуемых результатов.

Ключевые слова: инновации, мультипликатор инвестиций, акселератор инвестиций, экономическое развитие, инновационная продукция, обыкновенная продукция, экономическая безопасность.

\section{Введение}

Российская экономика, столкнувшаяся с активным внешним политическим и экономическим противодействием, а также с определенной волатильностью нефтяных цен, испытывает ряд достаточно серьезных трудностей. Так, реальные располагаемые денежные доходы населения падают четыре года подряд и только в августе 2018 г. по данным Росстата снизились на $0,9 \%$ по сравнению с августом 2017 г. В июле 2018 года индекс среднего чека россиян за один поход в магазин сократился до двухлетнего минимума. Сокращение расходов приводит к финансовым проблемам у производителей. Кроме проблем с доходами и, как следствие, финансами, производство (включая нефтяную промышленность) испытывает затруднения с доступом к современным технологиям и оборудованию. Это сказывается на качестве и номенклатуре продукции, внося свой негативный вклад в сложившуюся ситуацию.

В таких условиях импульсом для развития 
Российской Федерации и укрепления ее экономической безопасности должно стать увеличение производства качественной, востребованной продукции и создание вследствие этого дополнительных рабочих мест. Повышение спроса на рабочую силу и увеличение объема располагаемых доходов населения, которое, в свою очередь, инициирует вторую волну развития, связанную с ростом потребительского спроса.

Генерация такого импульса столкнется с санкционным противодействием, а также с достаточно жесткой конкуренцией со стороны ведущих мировых корпораций на основных рынках сбыта. Снижение курса национальной валюты дает ряд определенных преимуществ отечественным производителям, снижая стоимость их продукции по сравнению с ценами иностранных конкурентов. Но проблема производства продукции, соответствующей запросам современных покупателей и требуемым стандартам качества; продукции, способной конкурировать с производствами ведущих мировых корпораций, остается открытой.

Рецепт преодоления санкционного давления, конкуренции на основных мировых рынках сбыта и, как следствие, развития национальной экономики один - это перевод значительной части российской экономики на инновационный путь развития. Следствием чего станут два варианта развития ситуации:

1. Способность российских предприятий производить продукцию мирового уровня, выигрывая конкурентную борьбу у иностранных корпораций как на мировых, так и на отечественном рынках сбыта.

2. Создание новых рынков сбыта, еще не занятых иностранными конкурентами в результате производства новой продукции, ранее не представленной на рынках.

При этом второй вариант представляется более предпочтительным, так как дает отечественным предприятиям преимущества как производителям - первопроходцам на данном рынке.

Как указано выше, для решения поставленных задач требуется перевод российской экономики на инновационный путь развития, в связи с чем необходимо конкретизировать само понятие «инновации».

«Инновация возникает в результате использования результатов научных исследований и разработок, направленных на совершенствова- ние процесса производственной деятельности, экономических, правовых и социальных отношений в области науки, культуры, образования, в других сферах деятельности общества» [1].

Но далеко не любое нововведение является инновацией. «Инновация - это не всякое новшество или нововведение, а только такое, которое серьезно повышает эффективность действующей системы» [2].

Акцент в последнем определении стоит не только на повышении эффективности, но и на словах «действующая система». То есть инновацией признается нововведение, внедренное в действующую или вновь создаваемую, но, тем не менее, функционирующую систему. При этом нововведения могут быть внедрены как в производственной сфере, так и в финансовой, управленческой.

В том случае, если нововведение разработано, но не внедрено, его нельзя считать инновацией.

Согласно классификации «Руководства Осло» инновации делятся на 2 типа:

1. Поддерживающие - это инновации, которые развивают уже существующий и используемый способ производства продукта за счет усовершенствования и модернизации.

2. Прорывные - это инновации, которые производят кардинальную замену существующей и используемой технологии производства продукта (они нацелены на изменение привычного положения дел) [3].

Для того, чтобы сократить негативное влияние сильных международных конкурентов, Российской Федерации необходимо создавать новые рынки сбыта, а значит - сосредоточиться на прорывных инновациях.

\section{1. Инновации в экономике Российской} Федерации

Не смотря на определенные проблемы в российской науке и образовании (включая проблемы с финансированием и кадровым обеспечением), а также наличие трудностей в разработке и коммерциализации новых технологий, определенные качественные сдвиги в процессе инновационного развития экономики Российской Федерации есть. Одним из импульсов, приведшим к возникновению этих сдвигов, стали санкции, наложенные иностранными государствами на экономику Российской Федерации в последнее время. Именно санкции наиболее остро 
выявили технологическую зависимость российской экономики от стран Запада, угрожающую не только экономической, но и национальной безопасности страны.

Вопреки и благодаря санкциям Российская Федерация в последнее время смогла нарастить объемы производства инновационных товаров и услуг (табл. 1).

Темпы прироста объемов производства инновационной продукции в Российской Федерации представлены в табл. 2.

Анализируя табл. 2, можно сделать вывод, что производство инновационной продукции в Российской Федерации в 2017 г. по отношению к 2013 г. выросло на 18,79\%. Необходимо отметить негативный факт, заключающийся в том, что в 2017 г. наблюдается падение прироста инновационной продукции по сравнению с 2016 г. на
$4,52 \%$.

Также необходимо отметить тот факт, что данные в табл. 1 представлены в фактических ценах, поэтому при исключении влияния инфляции рост производства инновационной продукции будет более скромным. Но, с другой стороны, нельзя не отметить, что этот рост наблюдается в сложных условиях санкционного давления.

Для того, чтобы исключить инфляционную составляющую, рассчитаем долю инновационной продукции в общем объеме отгруженных товаров (табл. 3).

Из данных табл. 3 видно, что в последние годы доля инновационной продукции в общем объеме отгруженных товаров держалась на уровне 8,5\%, достигнув своего максимума в 2013 г. (9,15\%). В 2017 г. наблюдается определенное

Таблица 1. Объемы производства инновационных товаров и услуг в экономике Российской Федерации по отраслям, млрд. руб.[4]

\begin{tabular}{|l|c|c|c|c|c|c|c|}
\hline \multirow{2}{*}{ Вид экономической деятельности } & \multicolumn{7}{|c|}{ Год } \\
\cline { 2 - 9 } & 2011 & 2012 & 2013 & 2014 & 2015 & 2016 & 2017 \\
\hline Всего & 2106,7 & 2872,9 & 3507,9 & 3579,9 & 3843,4 & 4364,3 & 4167 \\
\hline в том числе & & & & & & & \\
\hline добыча полезных ископаемых & 516,8 & 522,9 & 523,2 & 648,5 & 368,4 & 420 & 489,4 \\
\hline обрабатывающие производства & 1309,2 & 1973,5 & 2518,6 & 2362,4 & 2856,3 & 3197 & 2832,8 \\
\hline $\begin{array}{l}\text { производство и распределение } \\
\text { электроэнергии, газа и воды }\end{array}$ & 21,4 & 13,2 & 30,7 & 26,5 & 33,6 & 106,7 & 80,8 \\
\hline связь & 49,5 & 41,4 & 31,5 & 30,6 & 39,5 & 53 & 74 \\
\hline $\begin{array}{l}\text { деятельность, связанная с использо- } \\
\text { ванием вычислитльной техники и } \\
\text { информационных технологий }\end{array}$ & 25 & 14,6 & 39,6 & 32,4 & 46,6 & 27,9 & 37,2 \\
\hline научные исследования и разработки & 159,4 & 284 & 344,7 & 464,2 & 482,3 & 517,9 & 620 \\
\hline
\end{tabular}

Таблица 2. Темпы прироста объемов производства инновационной продукции в Российской Федерации,\%

\begin{tabular}{|l|c|c|c|c|c|}
\hline \multirow{2}{*}{ Вид экономической деятельности } & \multicolumn{5}{|c|}{ Темп прироста } \\
\cline { 2 - 6 } & $\begin{array}{c}2014 \text { г. к } \\
2013 \text { г. }\end{array}$ & $\begin{array}{c}2015 \text { г. к } \\
2014 \text { г. }\end{array}$ & $\begin{array}{c}2016 \text { г. к } \\
2015 \text { г. }\end{array}$ & $\begin{array}{c}2017 \text { г. к } \\
2016 \text { г. }\end{array}$ & $\begin{array}{c}2017 \text { г. к } \\
2013 \text { г. }\end{array}$ \\
\hline Всего & 2,05 & 7,36 & 13,55 & $-4,52$ & 18,79 \\
\hline в том числе & & & & & $-6,46$ \\
\hline добыча полезных ископаемых & 23,95 & $-43,19$ & 14,01 & 16,52 & 12,48 \\
\hline обрабатывающие производства & $-6,2$ & 20,91 & 11,93 & $-11,39$ & 163,19 \\
\hline $\begin{array}{l}\text { производство и распределение } \\
\text { электроэнергии, газа и воды }\end{array}$ & $-13,68$ & 26,79 & 217,56 & $-24,27$ & 134,92 \\
\hline связь & $-2,86$ & 29,08 & 34,18 & 39,62 & $-6,06$ \\
\hline $\begin{array}{l}\text { деятельность, связанная с использо- } \\
\text { ванием вычислительной техники и } \\
\text { информационных технологий }\end{array}$ & $-18,18$ & 43,83 & $-40,13$ & 33,33 & 79,87 \\
\hline научные исследования и разработки & 34,67 & 3,9 & 7,38 & 19,71 & \\
\hline
\end{tabular}

Источник. Составлено авторами на основании данных табл. 1. 
Таблица 3. Расчет доли инновационной продукции в общем объеме отгруженных товаров [4]

\begin{tabular}{|l|c|c|c|c|c|c|c|c|}
\hline \multirow{2}{*}{ Отгружено товаров } & \multicolumn{7}{|c|}{ Год } \\
\cline { 2 - 10 } & 2010 & 2011 & 2012 & 2013 & 2014 & 2015 & 2016 & 2017 \\
\hline Всего, млрд.руб. & 25794,6 & 33407 & 35944,4 & 38334,5 & 41233,5 & 45525,1 & 51316,3 & 57611,1 \\
\hline $\begin{array}{l}\text { Инновационной продук- } \\
\text { ции, млрд. руб. }\end{array}$ & 1243,7 & 2106,7 & 2872,9 & 3507,9 & 3579,9 & 3843,4 & 4364,3 & 4167 \\
\hline $\begin{array}{l}\text { Доля инновационной про- } \\
\text { дукции, \% }\end{array}$ & 4,82 & 6,31 & 7,99 & 9,15 & 8,68 & 8,44 & 8,5 & 7,23 \\
\hline
\end{tabular}

снижение этой доли до 7,23\%. Но, с учетом того, что в 2010 г. доля инновационной продукции в общем объеме отгруженных товаров составила 4,82\%, а также с учетом того, что эта доля берется от растущего объема отгрузки товаров, темпы прироста которого превышают показатели инфляции, полученный результат с определенной осторожностью можно назвать положительным.

Российская экономика еще не избавилась от ресурсозависимости, проблемы, стоящие на пути инновационного развития, велики, но определённые сдвиги есть.

Мировая общественность также признает эти сдвиги. Так, за последние 8 лет Российской Федерации удалось значительно упрочить свои позиции в рейтингах, характеризующих условия экономического роста и его качество (Global Competitiveness Index, Doing Business, Global Innovation Index). Динамика значений этих индексов для Российской Федерации показана на рисунке 1.

Но при этом существуют и тревожные тенденции такие, как снижение производительности труда, устаревание и износ оборудования. Сохраняется чрезвычайно большое отставание от ведущих инновационных держав ЕС и Северной Америки. В результате проведенного анализа можно сделать вывод о том, что положительные сдвиги в ситуации с инновациями в экономике Российской Федерации есть, но положительная тенденция неустойчива и ее закрепление потребует значительных усилий, развития инновационного сектора экономики Российской Федерации. Подобные вывод подкрепляет и анализ динамики затрат на технологические инновации российских организаций, результаты которого показаны в табл. 4. Из данных табл. 4 видно значительное замедление темпов прироста затрат российских организаций на инновации в последние годы, начиная с 2014 г.

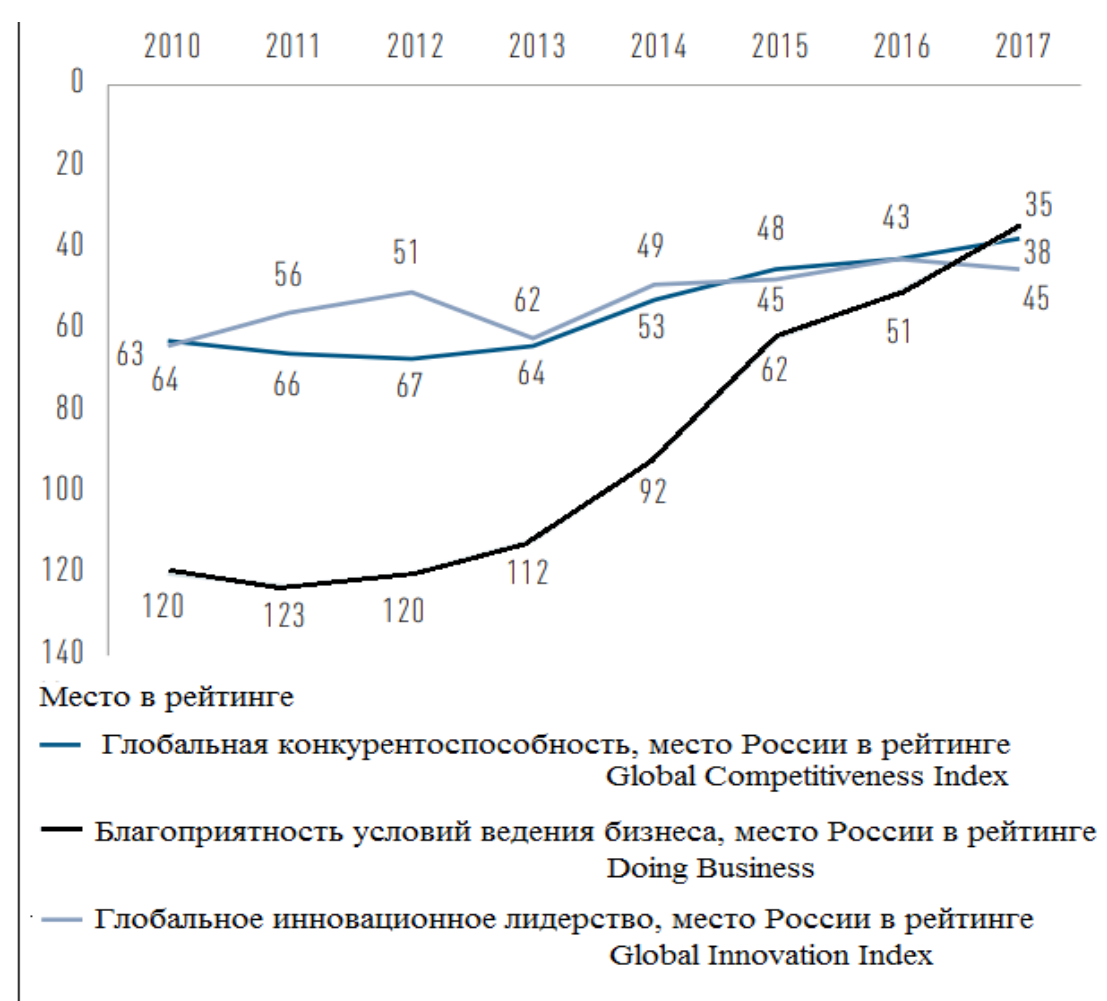

Puc. 1. Динамика позиций Российской Федерации в рейтингах Global Competitiveness Index, Doing Business, Global Innovation Index [5] 
Таблица 4. Затраты на технологические (продуктовые, процессные) инновации организаций [6]

\begin{tabular}{|l|c|c|c|c|c|c|c|c|}
\hline \multirow{2}{*}{ Показатель } & \multicolumn{7}{c|}{ Год } \\
\cline { 2 - 10 } & 2010 & 2011 & 2012 & 2013 & 2014 & 2015 & 2016 & 2017 \\
\hline Затраты на инновации, млрд. руб. & 400,8 & 733,8 & 904,6 & 1112,4 & 1211,9 & 1203,6 & 1284,6 & 1405,0 \\
\hline Темпы прироста к предыдущему году, \% & & 83,09 & 23,27 & 22,98 & 8,94 & $-0,68$ & 6,73 & 9,37 \\
\hline
\end{tabular}

2. Инновации как триггер мультипликативных эффектов

Инновационное развитие экономики Российской Федерации требует достаточно большого объема инвестиций. Необходимы инвестиции в развитие человеческого капитала (образование, наука, медицина), инвестиции в разработку новых технологий, разработку, создание и закупку современного инновационного оборудования, создание и развитие соответствующей инфраструктуры. Таким образом, инновационное развитие экономики Российской Федерации приведет к формированию достаточно объемных инвестиционных расходов. Ситуация осложняется тем, что эти расходы инициируют возникновение мультипликативных эффектов в экономике.

Мультипликатор инвестиций - коэффициент, показывающий, во сколько раз итоговый прирост валового национального дохода превышает исходный прирост инвестиций [7].

Следовательно, для решения задач по развитию инновационного сектора, необходимы инвестиции. Получатели этих инвестиций будут тратить их на закупку материалов, оборудования, наем персонала и т.п. Поставщики материалов, оборудования, а также персонал - для них инвестиционные траты будут доходом, часть которого они сберегут, а часть потратят на закупку потребительских и инвестиционных товаров, для продавцов которых эти траты также будут доходов, после чего процесс повторится заново.

Данный процесс не будет бесконечным, так как на каждом его этапе из этого процесса будут наблюдаться следующие оттоки финансовых ресурсов:

1. Часть ресурсов будет сберегаться каждым участником процесса (эту часть характеризует предельная склонность к сбережению).

2. Часть ресурсов будет изыматься государством в виде налогов, но они либо полностью, либо частично будут возвращены в экономику путем государственных расходов и инвестиций, каждая часть которых будет инициировать к жизни свои мультипликативные цепочки, что значительно усложняет анализируемый процесс.
3. Часть средств будет потрачена на приобретение материалов, оборудования и технологий за рубежом. В таком случае мультипликативная цепочка исчезает из экономического пространства Российской Федерации и проявляется в экономическом пространстве того иностранного государства, чью продукцию приобретут отечественные инвесторы и потребители. Мультипликативная цепочка может вернуться в отечественное экономическое пространство в том случае, если на определенном ее этапе иностранные производители закупят отечественную продукцию. Но даже в данном случае участок этой цепочки останется в иностранном экономическом пространстве, снижая объем экономического эффекта для Российской Федерации.

В том случае, если действующие производственные мощности будут не в состоянии удовлетворить весь объем дополнительного, мультипликативно увеличившегося спроса, возникнет необходимость создания дополнительных производственных мощностей. Что приведет к возникновению эффекта акселератора инвестиций.

Коэффициент акселератора показывает количество инвестиций, необходимое для создания дополнительного производства в объемах, способных удовлетворить дополнительный спрос [8].

Так как в основе действия механизма акселератора лежат новые инвестиции, его действие также будет создавать новые мультипликативные цепочки, которые снова будут создавать цепочки акселератора и т.д. Эти процессы достаточно сильно усложняют общую картину мультипликативных эффектов, инициированных инновациями, но они также будут конечными в связи с существованием вышеперечисленных оттоков финансовых ресурсов из этих процессов.

Развитие инновационного сектора в российской экономике инициирует широкий перечень положительных мультипликативных эффектов. К ним можно отнести следующие:

1. В результате действия мультипликативных эффектов прирост объемов национального 
производства, объемов национального дохода превысит запланированный, фактические темпы экономического роста будут выше запланированных.

2. Рост инновационных секторов экономики, вызванный инвестициями, выйдет за границы этих секторов и вызовет рост прочих секторов экономики в результате того, что инновационные секторы будут закупать у прочих необходимые ресурсы, оборудование и технологии в повышенном объеме.

3. Вследствие мультипликативного роста доходов будет наблюдаться диффузия инноваций, в результате которой инновации из региона-центра их развития будут распространяться на периферийные регионы, приводя к созданию на их территории новых производств, развитию инфраструктуры, что также будет сопровождаться возникновением эффектов мультипликатора - акселератора.

4. Взаимосвязь мультипликативных и инновационных процессов окажет значительное положительное влияние на развитие науки и образования. С одной стороны, развитие инновационного сектора потребует соответствующего кадрового обеспечения, что предъявит новые качественные требования к системе российского образования. С другой стороны, мультипликативный рост доходов граждан в результате развития инновационного сектора приведет к возникновению запроса со стороны граждан на более качественное образование для их детей. Что произойдет в рамках общего запроса на повышение качества жизни в связи с ростом дохода. Частью этого запроса будут повышенные требования потребителей к инфраструктуре, жилью, культурным и медицинским учреждения. Следовательно, генерируемый инновациями мультипликативный эффект будет иметь значительную социальную составляющую. Кроме этого конкуренция внутри мультипликативно развивающегося инновационного сектора приведет к росту спроса на научные кадры, научные исследования, в результате чего увеличится финансирование данной сферы в том числе - частным бизнесом.

5. В результате мультипликативного роста производства экономика получит дополнительные финансовые ресурсы для дальнейшего финансирования научных исследований, разработки инновационных технологий и продуктов.

6. Более благоприятный инвестиционный климат, сформировавшийся в результате роста инновационного сектора, привлечет иностранных инвесторов и стимулирует отечественных инвесторов в большем объеме вкладывать свои финансовые ресурсы в развитие национальной экономики. Последнее снизит отток финансовых ресурсов из экономики Российской Федерации за границу. Все вышеперечисленные процессы также будут генерировать мультипликативные эффекты. Необходимо отметить, что все это станет возможным, в том числе в результате благоприятных для инвестиционного климата Российской Федерации институциональных изменений.

7. В совокупности все вышеперечисленные мультипликативные эффекты, генерируемые развитием инновационного сектора, окажут значительное положительное влияние на экономическую и национальную безопасность страны.

3. Отрицательные мультипликативные эффекты как последствия развития инновационного сектора экономики

Если бы инновационный сектор экономики в процессе своего развития генерировал только положительные мультипликативные эффекты, развитие этого сектора было бы достаточно бурным и долговременным, а ограничивалось бы только текущим уровнем развития науки и технологий. Но в реальности чрезвычайно бурного и одновременно долговременного роста инновационного сектора экономики не наблюдается ни в одной стране мира. Одна из причин этого в том числе - отрицательные мультипликативные эффекты, генерируемые в процессе развития инновационного сектора.

В предыдущем пункте указано, что мультипликативные эффекты, возникшие в процессе развития инновационного сектора, приведут к росту национального дохода. Но это произойдет лишь в том случае, если экономика будет в состоянии удовлетворить дополнительный спрос, ставший результатом действия данных мультипликативных эффектов. В противном случае эти эффекты приведут к возникновению инфляции.

Также в предыдущем пункте статьи описано возникновение эффекта акселератора инвестиций в том случае, если экономика с учетом имеющихся в наличии производственных мощностей не способна удовлетворить дополнительный спрос, но способна создать новые мощности. Но новые, дополнительные мощности не 
могут быть созданы мгновенно. Следовательно, между мультипликативным приростом спроса и его удовлетворением будет наблюдаться временной разрыв, наличие которого приведет к возникновению инфляции.

Таким образом, негативным результатом действия мультипликативных эффектов, генерируемых развитием инновационного сектора может стать инфляция, иногда достаточно значительная. Что, в свою очередь приведет к сокращению объемов спроса, следовательно, вызовет необходимость сокращения производства, рост безработицы, падение доходов граждан и, как следствие приведет к новому сокращению объемов спроса. Таким образом, будет генерироваться отрицательный мультипликативный эффект, способный не только остановить развитие инновационного сектора экономики, но и повернуть это развитие вспять, приводя к его деградации.

Следовательно, положительные мультипликативные эффекты могут менять направления своего развития и перерождаться в отрицательные мультипликативные эффекты, оказывающие негативное влияние на динамику экономического развития.

Мультипликативный рост спроса на товары и ресурсы со стороны ряда непосредственно и опосредованно связанных между собой отраслей приведет к возникновению еще одного негативного эффекта. Отрасли неинновационного сектора экономики, получив от него импульс экономического роста (дополнительный доход), смогут приобретать ресурсы по более высоким ценам. Следовательно, достаточно велика вероятность того, что эти отрасли вступят в конкуренцию за ресурсы с инновационным сектором экономики. В результате этого процесс инновационного развития экономики может столкнуться с нехваткой необходимых ресурсов и, как минимум, удорожанием процесса реализации своих проектов, а как максимум - необходимостью частичного либо полного свертывания проектов в результате нехватки ресурсов и роста цен на них.

Описываемый эффект по своей сути является проявлением голландской болезни, предпосылкой которой служит «подъем одного из секторов экономики и значительное отставание в других» [9]. Но в данном случае голландская болезнь будет проистекать весьма специфично. Так как в случае с инновациями подъем одного из секторов экономики инициирует признаки голландской болезни не в нем самом, а в окружающих его секторах. Следовательно, в случае с взаимодействием развития инновационного сектора и мультипликативных эффектов имеет смысл говорить о голландской болезни, приводящей к возникновению своеобразного эффекта вытеснения (сокращение объема частных инвестиций при росте государственных расходов в результате повышения процентной ставки [10]).

Негативное действие мультипликативных эффектов, сгенерированных процессом развития инновационного сектора, будет заключаться и в том, что они (как положительные, так и отрицательные) усложнят процесс управления как развитием экономики государства в целом, так и развитием инновационного сектора в частности. Мультипликативные эффекты будут искажать намеченную траекторию развития инновационного сектора, приводя к поучению незапланированных результатов. Если квалификация управленческих кадров недостаточна для того, чтобы понять - какие именно план-факт отклонения вызваны действием мультипликативных эффектов и какие именно действия должны быть совершены для их устранения, могут быть приняты неправильные управленческие решения, усугубляющие ситуацию вплоть до возникновения масштабных кризисных явлений. Сложности добавит и тот факт, что любые управленческие решения, связанные с изменением объема инвестиционных расходов, будут генерировать собственные положительные и отрицательные мультипликативные эффекты.

4. Общая методика оценки мультипликативных эффектов, вызванных ростом объемов потребления инновационной продукции частным сектором

Для определения объема мультипликативных эффектов в современной экономике используется ряд формул. От достаточно простых, позволяющих рассчитать мультипликативный эффект с определенной степенью приближения (1) и (2)

$$
m=\frac{\Delta Y}{\Delta A}
$$

где $\mathrm{m}$ - величина мультипликатора автономных расходов;

$\Delta \mathrm{Y}$ - изменение равновесного ВНП;

$\Delta \mathrm{A}$ - изменение автономных расходов, неза- 
висимых от динамики дохода.

$$
M=\frac{1}{1-C_{y}}
$$

где $\mathrm{M}$ - величина мультипликатора инвестиций;

$\mathrm{C}_{Y}$ - величина предельной склонности к потреблению $\left(\mathrm{C}_{\mathrm{Y}}<1\right)$.

До более сложных, позволяющих учесть взаимодействие механизмов мультипликатора и акселератора инвестиций (3):

$$
M_{\text {обш }}=\frac{1}{\beta^{*}\left(1-\frac{\mu^{*}(1-\beta)}{\beta}\right)}
$$

где $\mathrm{M}_{\text {общ }}$ - величина мультипликатора, определяющего объем полного прироста спроса при взаимодействии отраслей (с учетом влияния акселератора инвестиций);

$\beta$ - предельная склонность к сбережению $(0 \leqslant \beta \leqslant 1)$;

$\mu$ - объем инвестиций, необходимый для производства одной единицы продукции (значение акселератора инвестиций) [7].

В связи с необходимостью определять величину мультипликативных эффектов, ввязанных именно с развитием инновационного сектора, общий подход к оценке мультипликативных эффектов, вызванных данным сектором, будет аналогичным при использовании разных формул оценки мультипликативных эффектов.

Необходимо разделить прирост продукции $(\Delta \mathrm{Y})$ на прирост инновационных продуктов $\left(\Delta \mathrm{Y}_{\mathrm{i}}\right)$ и прирост обычных продуктов $\left(\Delta \mathrm{Y}_{\text {об}}\right)$. И оценить вклад каждого из видов этого прироста в формирование общей величины мультипликатора автономных расходов. Общий объем прироста продукции будет определяться по формуле (4):

$$
\Delta Y=\Delta Y_{\text {об }}+\Delta \mathrm{Y}_{\mathrm{i}}
$$

С учетом того, что потребление в современной экономике складывается из потребления обычной и инновационной продукции, функция Кейнса (функция потребления) примет вид (5):

$$
C=C_{0}+C_{o \sigma} \times Y+C_{i} \times Y
$$

где C - общая величина потребления;

$\mathrm{C}_{0}$ - величина автономного, не зависящего от изменения объема дохода потребления;

$\mathrm{C}_{\text {об}}, \mathrm{C}_{\mathrm{i}}$ - предельные склонности к потребле- нию обыкновенной и инновационной продукции соответственно. $0<\left(\mathrm{C}_{\mathrm{oб}}+\mathrm{C}_{\mathrm{i}}\right)<1$.

Так как в состоянии равновесия должно выполняться следующее равенство (6):

$Y+\Delta Y=C+C_{\text {об }} \times(\mathrm{Y}+\Delta \mathrm{Y})+\mathrm{C}_{\mathrm{i}} \times(\mathrm{Y}+\Delta \mathrm{Y})+\mathrm{I}+\Delta \mathrm{I}$

где I - валовые внутренние частные инвестиции;

$\Delta \mathrm{I}-$ прирост валовых внутренних частных инвестиций.

Откуда:

$$
\Delta Y=\frac{\Delta I}{1-C_{\text {об }}-C_{i}}
$$

В таком случае величина мультипликатора с учетом спроса на инновационную продукцию $\left(\mathrm{M}_{\text {ин }}\right)$ может быть представлена как [11]:

$$
M_{u н}=\frac{1}{1-C_{\text {об }}-C_{i}}
$$

При этом сама величина прироста инвестиций $\Delta \mathrm{I}$ показанная в формуле (7), может быть разделена на величину прироста инвестиций в сектор производства обычной продукции $\Delta \mathrm{I}_{\text {об }}$ и величину прироста инвестиций в сектор производства инновационной продукции $\Delta \mathrm{I}_{\mathrm{i}}$. Следовательно, с учетом необходимости более точного учета экономических, мультипликативных последствий развития инновационного сектора, формула (7) примет вид (9):

$$
\Delta Y_{\text {об }}+\Delta Y_{i}=\frac{\Delta I_{\text {об }}+\Delta \mathrm{I}_{\mathrm{i}}}{1-C_{\text {об }}-C_{i}}
$$

Используя формулу (9) с помощью методов факторного анализа можно определить величину мультипликативного влияния на динамику национального продукта:

1. Прироста объема инвестиций в инновационный сектор экономики.

2. Величину изменения склонности к потреблению инновационной продукции.

Предложенная методика проиллюстрирована использованием достаточно простого варианта формулы мультипликатора, но, как указано выше, она может аналогично применяться для более сложных формул и методов оценки мультипликативных эффектов, порожденных развитием инновационного сектора в современной экономике. 


\section{Заключение}

Применение полученных результатов, прежде всего, позволит экономически стабилизировать процесс развития инновационного сектора экономики страны. В любом государстве инновационный сектор достаточно нестабилен - периоды бурного, взрывного развития могут сменяться периодами застоя и упадка. Инновационный бум может приводить к возникновению на бирже финансовых «пузырей», схлопывание которых оказывает значительное депрессивное влияние не только на динамику инновационного сектора, но и на общую динамику экономической конъюнктуры. Нестабильности развитию инновационного сектора добавляет и неравномерность развития науки, когда прорывные открытия, дающие значительный импульс инновационному развитию, могут сменяться длительными периодами научного поиска.

Мультипликативные эффекты, как указано выше, вносят свой вклад в формирование нестабильности анализируемых процессов, изменяя траекторию их развития, приводя к возникновению дополнительного спроса, провоцируя инфляцию. В результате действия этих эффектов целенаправленное развитие инновационного сектора экономики может привести к получению незапланированных результатов. Даже в том случае, если они будут положительными, это усложнит процессы планирования, контроля над выполнением планов для разных предприятий, отраслей и секторов экономики.

Внедрение методов оценки объема и влияния мультипликативных эффектов в процесс принятия управленческих решений позволит сделать процесс управления более обоснованным, а его результаты более предсказуемыми, что снизит уровень риска, который в процессе развития инновационного сектора итак достаточно высок вследствие специфики инновационного производства и инновационной продукции.

Работа выполнена при финансовой поддержке РФФИ в рамках научного проекта № 16-0200658-ОГН/18.

\section{Библиографический список}

1. Глаголев С.Н. Дорошенко Ю.А., Моисеев В.В. Актуальные проблемы инвестиций и инноваций в современной России. Монография. 2014. 425 с.

2. Милославский И. С. Новизна с последствиями [Электронный ресурс] // Газета «Известия». 2009. URL: http:// www.izvestia.ru/news/349367 (дата обращения 07.11.2018)

3. Руководство Осло. Рекомендации по сбору и анализу инновационных данных. Совместная публикация ОЭСР и Евростата. Москва. 2010. 107 с.

4. Росстат. Официальная статистика. Наука и инновации [Электронный ресурc] URL: http://www.gks.ru/wps/ wcm/connect/rosstat_main/rosstat/ru/statistics/science/\# (дата обращения 07.11.2018)

5. Министерство экономического развития Российской Федерации. Национальный доклад об инновациях в России 2017 [Электронный ресурс] URL: http://www.rvc.ru/upload/iblock/c64/RVK_innovation_2017.pdf

6. Росстат. Официальная статистика. Затраты на технологические (продуктовые, процессные) инновации организаций URL: http://www.gks.ru/free_doc/new_site/business/nauka/innov-n5.xls (дата обращения 07.11.2018)

7. Еремин В.В. Анализ механизма взаимодействия мультипликатора и акселератора инвестиций как процесса в рядах динамики // Экономика. Предпринимательство. Окружающая среда. Москва.2014. № 4 (60). С 26-33.

8. Еремин В.В. Математический анализ мультипликатора автономных расходов в статике и динамике. Монография. Уфа. 2015. 172 с.

9. Сухенко А.В. Семенова Э.Н. «Голландская болезнь» российской экономики и специфика ее проявления // Студенческая наука XXI века. Чебоксары. 2015. С. 379-383.

10. Roger W. Spencer \& William P. Yohe, 1970. «The «Crowding Out» of Private Expenditures by Fiscal Policy Actions», Federal Reserve Bank of St. Louis Review, October, P. 12-24.

11. Горшков А.П. Мультипликационные эффекты, индуцированные частным сектором при производстве инновационной продукции // Вестник Пермского национального исследовательского политехнического университета. Социально-экономические науки. 2018. № 2. С. 156-165. 